'Departamento de

Medicina, Escuela de

Medicina, Universidad de

Valparaíso, Valparaíso,

Chile.

${ }^{2}$ Servicio de Medicina, Sección de Nefrología, Hospital Dr. Gustavo Fricke, Viña del Mar, Chile.

${ }^{3}$ Servicio de Medicina,

Sección de Nefrología,

Hospital Naval A. Nef, Viña

del Mar, Chile.

${ }^{4}$ Servicio de Radiología,

Hospital Naval A. Nef, Viña

del Mar, Chile.

${ }^{5}$ Departamento de

Anatomía Patológica,

Facultad de Medicina,

Pontificia Universidad

Católica de Chile,

Santiago, Chile.

Recibido el 27 de febrero de 2012, aceptado el 23 de julio de 2012.

Correspondencia a:

Dr. Jorge Vega

5 Norte 1035, Viña del

Mar, Chile.

Fax: 56-32-2974237.

E-mail: jvegastieb@gmail.

com

\section{Remisión completa de síndrome nefrótico asociado a carcinoma cervicouterino luego de tratamiento del tumor. Comunicación de 2 casos}

\author{
JORGE VEGA ${ }^{1,2,3}$, MARIO SANTAMARINA ${ }^{4}$, GONZALO P. MÉNDEZ ${ }^{5}$.
}

\section{Complete remission of nephrotic syndrome associated with carcinoma of the cervix after treatment of the tumor. Report of two cases}

Nephrotic syndrome secondary to paraneoplastic glomerulopathies is exceptional. We are aware of only three cases reported of cervical carcinomas associated with nephrotic syndrome. Two women, aged 40 and 79 years, presented with nephrotic syndrome. The first had a membranous nephropathy and the second was not biopsied. The first women had a metrorrhagia after 8 months of unsuccessful therapy with corticosteroids and immunosuppressive drugs. An advanced cervical carcinoma with lymph node metastases was found. In the second patient, a cervical carcinoma and hematometra was discovered two months after diagnosis of a nephrotic syndrome. The syndrome subsided completely, nine months after radiotherapy and chemotherapy in the first patient and 10 months after hysterectomy in the second patient.

(Rev Med Chile 2012; 140: 1580-1584).

Key words: Chemoradiotherapy; Chemotherapy, adjuvant; Nephrotic syndrome; Uterine cervical neoplasms.
L os tumores ginecológicos pueden presentarse como un síndrome paraneoplásico (SPN). Sus manifestaciones pueden ser diversas, incluyendo al síndrome nefrótico $(\mathrm{SN})^{1-8}$. Los $\mathrm{SN}$ asociados a carcinoma cérvico-uterino (CCU) son muy raros, existiendo 3 casos comunicados en la literatura $^{8-10}$. Presentamos 2 casos de síndrome nefrótico paraneoplásico (SNPNP) asociado a un CCU.

\section{Casos clínicos}

\section{Caso 1}

Mujer de 40 años que 5 meses después de su tercer parto (noviembre de 2007) presentó edema facial y de extremidades. La presión arterial era normal. La albuminemia fue $1,40 \mathrm{~g} /$ dl, colesterol $562 \mathrm{mg} / \mathrm{dl}$, triglicéridos $716 \mathrm{mg} / \mathrm{dl}$, creatinina 1,0 mg/dl, nitrógeno ureico (NU) 10,7 $\mathrm{mg} / \mathrm{dl}$, proteinuria $20.630 \mathrm{mg} / 24 \mathrm{~h}$, clearance de creatinina $69 \mathrm{ml} / \mathrm{min} / 1,73 \mathrm{~m}^{2}$ y el sedimento de orina normal. Existía un hipotiroidismo subclínico (TSH 9,63 microUI/ml). Los ANA, ANCA, ASLO, C'3 y C'4 fueron negativos o normales. Se administraron 3 pulsos de metilprednisolona continuando con prednisona oral (60 mg/día). La biopsia renal mostró una nefropatía membranosa (NM) (Figura 1 ). Se adicionó ciclofosfamida (CF), administrándola en pulsos mensuales de $500 \mathrm{mg}$, enalapril y tiroxina. Seis meses después (mayo de 2008), el SN persistía incólume (proteinuria $18.790 \mathrm{mg} /$ día, albuminemia $1,9 \mathrm{~g} / \mathrm{dl}$, colesterol $321 \mathrm{mg} / \mathrm{dl}$, triglicéridos $429 \mathrm{mg} / \mathrm{dl}$ ), por lo que se cambió la CF por micofenolato mofetil (MMF) $2 \mathrm{~g} /$ día, manteniendo la prednisona. A los 8 meses del inicio de la terapia (julio de 2008) presentó 


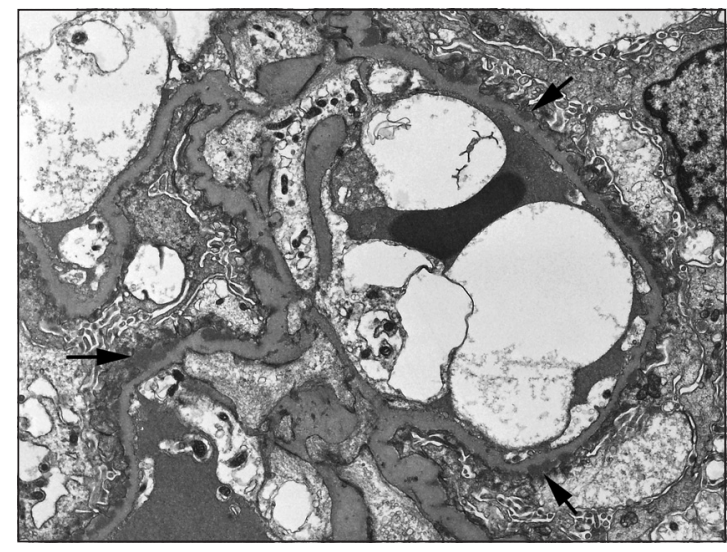

Figura 1. Microscopía electrónica (caso 1). Segmento de ovillo glomerular que incluye tres asas capilares. Se reconocen numerosos depósitos electron-densos subepiteliales (flechas), finamente granulares y confluentes, compatibles con distribución de tipo membranoso (acetato de uranilo-tetróxido de osmio; x8.000).

metrorragia. El examen ginecológico mostró una masa en el cuello uterino correspondiendo a un CCU epidermoide con linfangitis carcinomatosa. La tomografía mostró adenopatías pelvianas y retroperitoneales, etapificándose el cáncer en como FIGO III AB. Se suspendió el MMF. Se descartó practicar una histerectomía por lo avanzado del cáncer y se efectuó radioterapia externa y quimioterapia con cisplatino. En enero de 2009, 6 meses después del diagnóstico del CCU, mientras recibía braquiterapia, prednisona $20 \mathrm{mg}$, lovastatina, enalapril y tiroxina; el síndrome nefrótico era moderado (albuminemia 3,8 g/dl, colesterol 254 $\mathrm{mg} / \mathrm{dl}$, triglicéridos $352 \mathrm{mg} / \mathrm{dl}$, proteinuria 3.700 $\mathrm{mg} / 24 \mathrm{~h}$ ), existía hematuria microscópica y no había azotemia. Al año del diagnóstico del CCU (julio de 2009), el SN estaba en remisión completa. En los 2 años siguientes presentó una tromboflebitis iliofemoral, algunos episodios de cistitis y aparecieron adenopatías cervicales y supraclaviculares izquierdas metastásicas, que motivaron una nueva quimioterapia. En noviembre de 2011, cuatro años después del diagnóstico del SN, su estado general era bueno a pesar de sus metástasis y el síndrome nefrótico persistía en remisión completa.

\section{Caso 2}

Mujer de 79 años, hipertensa, que consultó por edema de extremidades inferiores en junio de 2010. Existía hematuria microscópica y proteinuria de $1.776 \mathrm{mg} / 24 \mathrm{~h}, \mathrm{NU} 46,6 \mathrm{mg} / \mathrm{dl}$, creatininina $1,6 \mathrm{mg} / \mathrm{dl}$, clearance de creatinina 42 $\mathrm{ml} / \mathrm{min} / 1,73 \mathrm{~m}^{2}$, proteinemia $6,8 \mathrm{~g} / \mathrm{dl}$, albuminemia 4,3 g/dl, colesterol $290 \mathrm{mg} / \mathrm{dl}$ y triglicéridos $155 \mathrm{mg} / \mathrm{dl}$. Se prescribió furosemide, enalapril y atorvastatina. Reapareció 7 meses después (enero de 2011) en anasarca. La proteinuria fue 9.614 $\mathrm{mg} / 24 \mathrm{~h}$, proteinemia $4,7 \mathrm{~g} / \mathrm{dl}$, albuminemia 2,3 $\mathrm{g} / \mathrm{dl}$, creatinina $2,1 \mathrm{mg} / \mathrm{dl}$, clearance de creatinina $38,6 \mathrm{ml} / \mathrm{min} / 1,73 \mathrm{~m}^{2}$ ) y el examen de orina mostró hematuria. La ecotomografía describió un riñón derecho de 9,6 x 6,1 cm y el izquierdo de 10,5 x $5,7 \mathrm{~cm}$ con aumento difuso de la ecogenicidad y diferenciación córtico-medular conservada. El complemento C'3 y C'4 fue normal, la serología viral (virus B, C, HIV) y de autoinmunidad (ANA, ANCA, ENA, antiDNA) fueron negativas y no se detectaron paraproteínas. Se prescribió diuréticos y se le indicó una biopsia renal. En la hospitalización motivada para efectuar la biopsia renal se constató que la función renal había empeorado notoriamente (creatinina 5,5 mg/dl, NU $66 \mathrm{mg} / \mathrm{dl}$ ) por lo que se desistió de efectuar la biopsia e instaló un catéter iniciándose hemodiálisis. Un mes después, la anorexia pertinaz y una baja de peso progresiva hicieron sospechar un cáncer. La tomografía mostró una imagen hipodensa de $3,3 \mathrm{~cm}$ a nivel del cérvix que se reforzaba con el medio de contraste y una dilatación de la cavidad endometrial (Figura 2). Una ecotomografía transvaginal mostró una cavidad uterina ocupada por líquido que hizo sospechar una colección hemática. Se efectuó histerectomía y anexectomía bilateral. Existía una lesión que obstruía el canal cervical y que correspondía a un carcinoma epidermoide con permeación a los linfáticos vecinos. Por sus malas condiciones generales la familia decidió no hacer quimioterapia ni radioterapia. Dos meses después (mayo de 2011) se infectó el catéter de diálisis y se retiró. Persistía el SN y había una leve reducción de la azotemia (creatinina 4,95 mg/dl, NU $72 \mathrm{mg} /$ dl), por lo que se suspendió temporalmente la diálisis, antes de instalar un nuevo catéter. En julio de 2011, se constató mejoría en la función renal (creatinina 1,77 mg/dl, clearance de creatinina 30,2 $\mathrm{ml} / \mathrm{min} / 1,73 \mathrm{~m}^{2}$ ) y remisión parcial del síndrome nefrótico (proteinuria $1.603 \mathrm{mg} / 24 \mathrm{~h}$, proteinemia $5,46 \mathrm{~g} / \mathrm{dl}$, colesterol $229 \mathrm{mg} / \mathrm{dl}$, triglicéridos 116 $\mathrm{mg} / \mathrm{dl}$ ). En los meses siguientes la proteinuria continuó reduciéndose y en su último control (enero de 2012) la paciente estaba asintomática y el SN en remisión completa. 


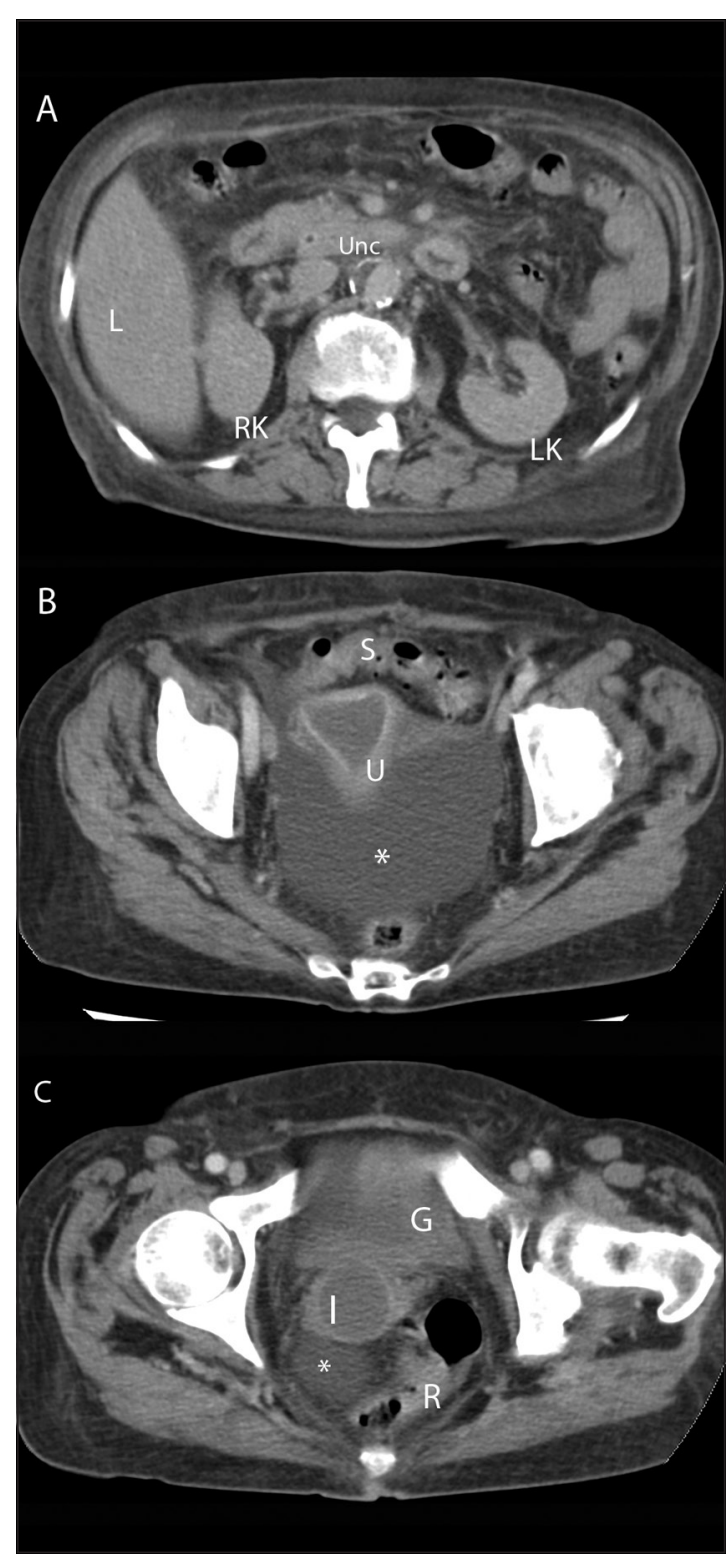

Figura 2. Imágenes axiales de tomografía computada con contraste ev. A. Imagen tardía (4 min) después de la administración de medio de contraste endovenoso, donde se evidencia retardo en la concentración y excreción del medio de contraste en ambos riñones. Se evidencia además, aumento de la densidad de la grasa de cavidad peritoneal y del tejido celular subcutáneo. RK: riñón derecho; LK: riñón izquierdo; L: hígado; unc: proceso uncinado del páncreas; B y C. Imágenes obtenidas de la pelvis que muestran la presencia de una cavidad endometrial marcadamente distendida, secundariamente a obstrucción del canal cervical por un cáncer de cuello uterino. Presencia de líquido ascítico (asterisco). El colon sigmoides (S) presenta divertículos sin complicación. La vejiga (G) presenta escasa repleción; (U): útero; (I): istmo uterino.

\section{Discusión}

Las glomerulopatías paraneoplásicas (GPNP) son originadas por productos de las células tumorales: hormonas, factores de crecimiento, citoquinas o antígenos tumorales ${ }^{1,11}$.

Los tipos histológicos comunicados más frecuentemente son la NM y la nefropatía de cambios mínimos (NCM). La NM generalmente se asocia a carcinomas sólidos y la NCM a procesos linfoproliferativos ${ }^{10-12}$. Se han descrito con menor frecuencia glomerulonefritis (GN) por IgA, GN mesangiocapilar, gloméruloesclerosis focal y segmentaria, GN crecéntica, amiloidosis y microangiopatía trombótica ${ }^{12,13}$.

Los cánceres más comúnmente asociados a NM son broncopulmonares y gástricos. Con menor frecuencia: renales, prostáticos, colorrectales, mamarios, pancreáticos, tímicos, etc ${ }^{12,13}$. Es extremadamente raro que un $\mathrm{SN}$ se asocie a tumores ginecológicos ${ }^{1,11,12,14}$. En estas 2 pacientes el tumor fue un carcinoma invasor de células escamosas del cuello uterino.

Se debe sospechar que la NM puede corresponder a una GPNP en las siguientes situaciones: a) cuando el paciente es mayor de 60 años o fumador; b) cuando no existe una etiología evidente (Ej: infecciones, drogas, enfermedades colágenovasculares); c) cuando existe una relación temporal entre el diagnóstico del SN y el cáncer (hasta 12 meses); d) cuando el SN remite al tratar al cáncer; e) cuando el SN recurre al reaparecer el cáncer; f) cuando existen $>8$ células inflamatorias en un glomérulo; g) cuando existe deterioro de la función renal al diagnóstico de $\mathrm{SN}^{10-12}$.

En una de las pacientes de esta comunicación se sospechó que el SN podía estar asociado a un cáncer debido a la persistencia de una anorexia pertinaz, un mes después de haber ingresado a diálisis, en la otra enferma el cáncer se descubrió en la investigación de una metrorragia.

En series de pacientes con NM se ha encontrado que la proporción asociada a cánceres es 10\% (rango: 5\%-22\%) ) $^{10,14}$. Ella aumenta en pacientes mayores de 60 años ${ }^{10,11,15,16}$. Los portadores de una NM tienen una prevalencia de cáncer 5 a 10 veces mayor que la población de referencia ${ }^{10,17}$ y tienen un riesgo de cáncer 2 a 3 veces mayor en los 4 años siguientes $^{12}$. La patogénesis de la NM asociada a cáncer no está dilucidada. Se ha planteado que la NM pudiera producirse por el depósito en el 
glomérulo de complejos inmunes circulantes (en que el antígeno proviene del tumor), la formación de complejos inmunes "in situ" al existir antígenos tumorales plantados en el glomérulo, o que estructuras glomerulares propias se tornan inmunogénicas por perturbaciones en la inmunidad originadas por el tumor ${ }^{11}$. Los pacientes con cánceres están expuestos a una antigenemia continua (antígenos tumorales, re-expresión de antígenos fetales, antígenos virales o antígenos autólogos de autoinmunidad $)^{18}$. Antígenos tumorales como el antígeno carcinoembrionario, antígeno prostático específico y antígenos de melanoma se han encontrado en asociación a GPNP, sin embargo, su rol causal no se ha demostrado ${ }^{13}$. Excepcionalmente se han recuperado antígenos tumorales en los depósitos existentes en los glomérulos ${ }^{9,20}$. Se ha postulado que el depósito de las diferentes subclases de IgG en la membrana basal glomerular es diferente en las NM idiopáticas respecto a las NM PNP y que ello pudiera orientar en la sospecha de la existencia de un cáncer asociado al $\mathrm{SN}^{21}$. En sólo una de nuestras pacientes se efectuó una biopsia renal, que mostró una NM. En la restante, que no fue biopsiada, es probable que también se tratara de una NM aunque no es descartable que se haya tratado de otro tipo histológico, como NCM.

En $40 \%$ a $45 \%$ de los pacientes el SN se presenta antes que se diagnostique el cáncer, en $40 \%$ el diagnóstico de ambas patologías es simultáneo y en el 20\% restante, la aparición del SN es posterior al diagnóstico del cáncer ${ }^{14}$. En la gran mayoría de los pacientes ambos fenómenos se presentan dentro de un período menor de 12 meses. En las 2 enfermas comunicadas el SN se presentó antes del diagnóstico del tumor ( 2 y 8 meses) lo que también ocurrió en los 3 casos de CCU comunicados previamente ${ }^{8-10}$.

La mayoría de los pacientes con GPNP permanecen nefróticos hasta que fallecen. La muerte habitualmente es secundaria al cáncer pero algunos fallecen por una complicación de la nefropatía ${ }^{10}$. La sobrevida promedio después del diagnóstico del SN es 12 meses y después del diagnóstico de cáncer 3 meses $^{14}$. Algunos pacientes deterioran su función renal pero es excepcional que ingresen a diálisis ${ }^{10,14}$. Una de nuestras pacientes ingresó a diálisis por una rápida progresión de su insuficiencia renal, sin embargo, después de la extirpación del útero tumoral, la función renal se recuperó parcialmente pudiendo retirarse de diálisis.
La remisión del SN se observa sólo en los pacientes que han obtenido el control de su cáncer con el tratamiento empleado. Ello ha sido un fuerte argumento para sostener la relación causal entre la presencia del cáncer y el $\mathrm{SN}^{10}$. Rara vez se ha practicado una biopsia renal para conocer si la remisión clínica se asocia a una remisión histológica $^{12}$. Existe también una proporción de enfermos en que la proteinuria continúa a pesar de obtenerse la remisión del cáncer. Sin embargo, cuando el cáncer no es controlado con el tratamiento, el SN persiste ${ }^{10}$. También cuando ocurre una recaída del tumor puede producirse una recaída del SN, si bien ambas entidades pueden evolucionar por separado y no reaparecer la proteinuria a pesar de la reaparición del tumor o sus metástasis ${ }^{12}$. En nuestras 2 enfermas se observó la remisión completa del SN después del tratamiento del CCU. Ello ocurrió 9 meses y 10 meses, respectivamente. Una de las enfermas presentó posteriormente metástasis ganglionares cervicales y supraclaviculares, sin embargo, el SN no recurrió.

Es frecuente que el SNPNP inicialmente sea considerado como originado en una GN primaria. Recientemente se identificó el antígeno al cual están dirigidos los anticuerpos en la NM primaria y que corresponde al receptor de la Fosfolipasa A2 ubicado en el podocito. Los anticuerpos de la subclase IgG4 pueden encontrarse hasta en el 80\% de los pacientes ${ }^{22}$. Estos anticuerpos también se han encontrado en una proporción de GNPNP ${ }^{23}$. La prescripción de terapia inmunosupresora por la presunción de que la NM es idiopática puede perjudicar al paciente, ya que además de inefectiva puede ser dañina, acelerando la progresión del cáncer ${ }^{13}$. Es probable que ello haya sucedido en la primera paciente, la que además de corticoides recibió ciclofosfamida y MMF. El tratamiento de la GPNP debe ir dirigido al cáncer ${ }^{12}$.

Si bien, la existencia de una neoplasia maligna ocurre en aproximadamente $10 \%$ de los portadores de una NM, parece prudente descartar razonablemente la existencia de un cáncer, especialmente en los pacientes con factores de riesgo, antes de iniciar terapia inmunosupresora.

\section{Referencias}

1. Ashour AA, Verschraegen CF, Kudelka AP, Kavanagh JJ. Paraneoplastic syndromes of gynecologic neoplasms. J Clin Oncol 1997 15: 1272-82. 
2. Young RH, Scully RE, McCluskey RT. A distinctive glomerular lesion complicating placental site trophoblastic tumor: report of two cases. Hum Pathol. 1985; 16: 35-42.

3. Hoyt RE, Hamilton JF. Ovarian cancer associated with the nephrotic syndrome. Obstet Gynecol 1987; 70: 513 4.

4. Freistadt H, Fine MN, Roth RE. Nephrotic syndrome associated with degenerating uterine leiomyoma. West J Med 1984; 141: 381-2.

5. Bashour BN, Mancer K, Rance CP. Malignant mixed mullerian tumor of the cervix following cyclophosphamide therapy for nephrotic syndrome. J Pediatr 1973; 82: 292-3.

6. Kijima Y, Yoshinaka H, Owaki T, Nozaki T, Hamada T, Yasumoto $\mathrm{Y}$, et al. Breast cancer with nephrotic syndrome: report of two cases. Surg Today 2004; 34: 755-9.

7. Meydanli MM, Erguvan R, Altinok MT, Ataoglu O, Kafkasli A. Rare case of neuroendocrine small cell carcinoma of the endometrium with paraneoplastic membranous glomerulonephritis. Tumori 2003; 89: 213-7.

8. Ito C, Akimoto T, Nakazawa E, Komori S, Sugase T, Chinda J, et al. A case of cervical cancer-related membranous nephropathy treated with radiation therapy. Intern Med. 2011; 50: 47-51.

9. Lee JC, Yamauchi H, Hopper J Jr. The association of cancer and the nephrotic syndrome. Ann Intern Med 1966; 64: 41-51.

10. Lefaucheur C, Stengel B, Nochy D, Martel P, Hill GS, Jacquot C, et al; GN-PROGRESS Study Group. Membranous nephropathy and cancer: Epidemiologic evidence and determinants of high-risk cancer association. Kidney Int. 2006; 70: 1510-7.

11. Ronco PM. Paraneoplastic glomerulopathies: new insights into an old entity. Kidney Int. 1999; 56: 355-77.

12. Bacchetta J, Juillard L, Cochat P, Droz JP. Paraneoplastic glomerular diseases and malignancies. Crit Rev Oncol Hematol. 2009; 70: 39-58.
13. Lien YH, Lai LW. Pathogenesis, diagnosis and management of paraneoplastic glomerulonephritis. Nat Rev Nephrol. 2011; 7: 85-95.

14. Burstein DM, Korbet SM, Schwartz MM. Membranous glomerulonephritis and malignancy. Am J Kidney Dis 1993; 22: 5-10.

15. Zech P, Colon S, Pointet P, Deteix P, Labeeuw M, Leitienne $\mathrm{P}$. The nephrotic syndrome in adults aged over 60: etiology, evolution and treatment of 76 cases. Clin Nephrol 1982; 17: 232-6.

16. Eagen JW. Glomerulopathies of neoplasia. Kidney Int 1977; 11: 297-303.

17. Brueggemeyer CD, Ramírez G. Membranous nephropathy: a concern for malignancy. Am J Kidney Dis 1987; 9: 23-6.

18. Norris SH. Paraneoplastic glomerulopathies. Semin Nephrol 1993; 13: 258-72.

19. Wakashin M, Wakashin Y, Iesato K, Ueda S, Mori Y, Tsuchida $\mathrm{H}$, et al. Association of gastric cancer and nephrotic syndrome. An immunologic study in three patients. Gastroenterology 1980; 78: 749-56.

20. Couser WG, Wagonfeld JB, Spargo BH, Lewis EJ. Glomerular deposition of tumor antigen in membranous nephropathy associated with colonic carcinoma. Am J Med. 1974; 57: 962-70.

21. Ohtani H, Wakui H, Komatsuda A, Okuyama S, Masai $\mathrm{R}$, Maki N, et al. Distribution of glomerular IgG subclass deposits in malignancy-associated membranous nephropathy. Nephrol Dial Transplant. 2004; 19: 574-9.

22. Beck LH Jr, Bonegio RG, Lambeau G, Beck DM, Powell DW, Cummins TD, et al. M-type phospholipase A2 receptor as target in idiopathic membranous nephropathy. N Engl J Med 2009; 361: 11-21.

23. Qin W, Beck LH Jr, Zeng C, Chen Z, Li S, Zuo K, et al. Anti-phospholipase A2 receptor antibody in membranous nephropathy. J Am Soc Nephrol 2011; 22: 1137 43. 